\title{
Coupling Quantum Emitters to Random 2D Nanoplasmonic Structures
}

\author{
Thomas A. R. Purcell, ${ }^{1}$ Matan Galanty, ${ }^{2}$ Shira Yochelis, ${ }^{2}$ Yossi Paltiel ${ }^{2}$ and Tamar \\ Seideman $^{1 *}$
}

${ }^{1}$ Chemistry Department, Northwestern University, Evanston, IL 60208

${ }^{2}$ Applied Physics Department and the Center for Nanoscience and Nanothechnology, The Hebrew University of Jerusalem, Jerusalem 91904, Israel

*Corresponding Author e-mail: t-seideman@northwestern.edu Telephone: 1 (847) 467-4979 


\section{Parameters for CdSe and PbS QD Dielectric Functions}

Both quantum dot (QD) dielectric functions are approximated by a single Lorentzian. The high frequency dielectric constant was taken to be that of the bulk semiconductor for both the CdSe $(610 \mathrm{~nm})$ and $\mathrm{PbS}(850 \mathrm{~nm})$ quantum dots. The pole parameters used for the CdSe dielectric function are $\omega_{Q D}=2.03 \mathrm{eV}, \gamma_{Q D}=140 \mathrm{meV}$, and $\sigma=0.65$. The PbS parameters are $\omega_{Q D}=1.46$ $\mathrm{eV}, \gamma_{Q D}=260 \mathrm{meV}$, and $\sigma=5.00$. Figure $\mathrm{S} 1$ shows the absorption cross-section of both QDs.

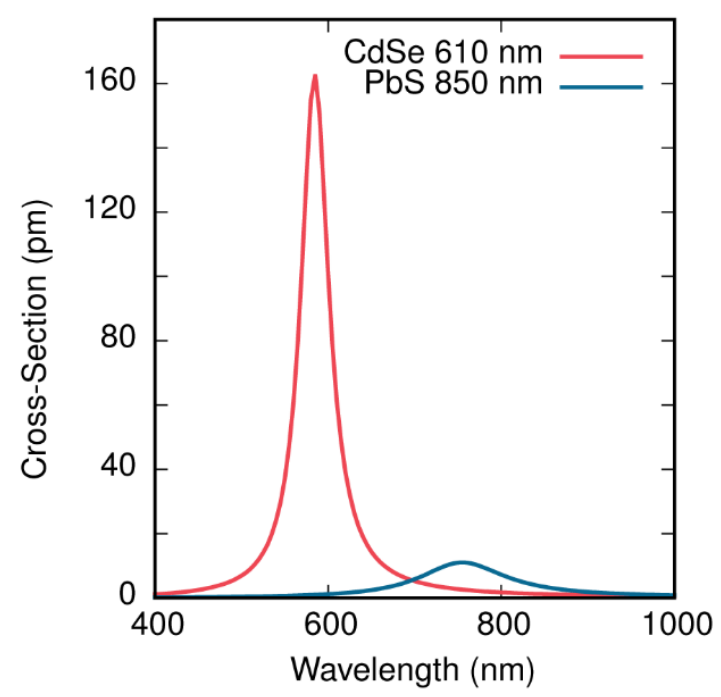

Figure S1: Absorption cross-section of the CdSe $610 \mathrm{~nm}$ QD and the PbS $850 \mathrm{~nm}$ QD calculated within the Lorentz-Drude dielectric model.

\section{FDTD Cell}

Figure S2 shows a diagram of the FDTD cell. The nanoparticle system is in the center of the cell, with the total-field scattered-field (TFSF) region placed $20 \mathrm{~nm}$ away from the gold nanoellipse at all sides. The system is excited using a Gaussian pulse with a center frequency of $480 \mathrm{THz}$ and a pulse duration of 1.39 fs propagating from the bottom of the TFSF region represented by the red box. The enhancement factor calculations are preformed using a $2 \AA$ step size, while the other CdSe 610 nm QD and PbS 850 nm QD calculations used a $5 \AA$ and a $2.5 \AA$ step size respectively. The time step $(\Delta t)$ is set by

$$
\Delta t=\frac{\Delta x}{c S}
$$


where $\Delta x$ is the spatial step size, $c$ is the speed of light, and $\mathrm{S}$ is the Courant factor, set to 0.5 for all calculations. The absorption and scattering cross-sections are calculated in the regions shown in the blue boxes $10 \mathrm{~nm}$ inside and outside the TFSF region respectively. The QD absorption cross-section is calculated in a region surrounding the QD with no interaction with the gold nanoislands. The cell is terminated by a convolution perfectly matched layer that is at least 20 grid points wide in all directions.

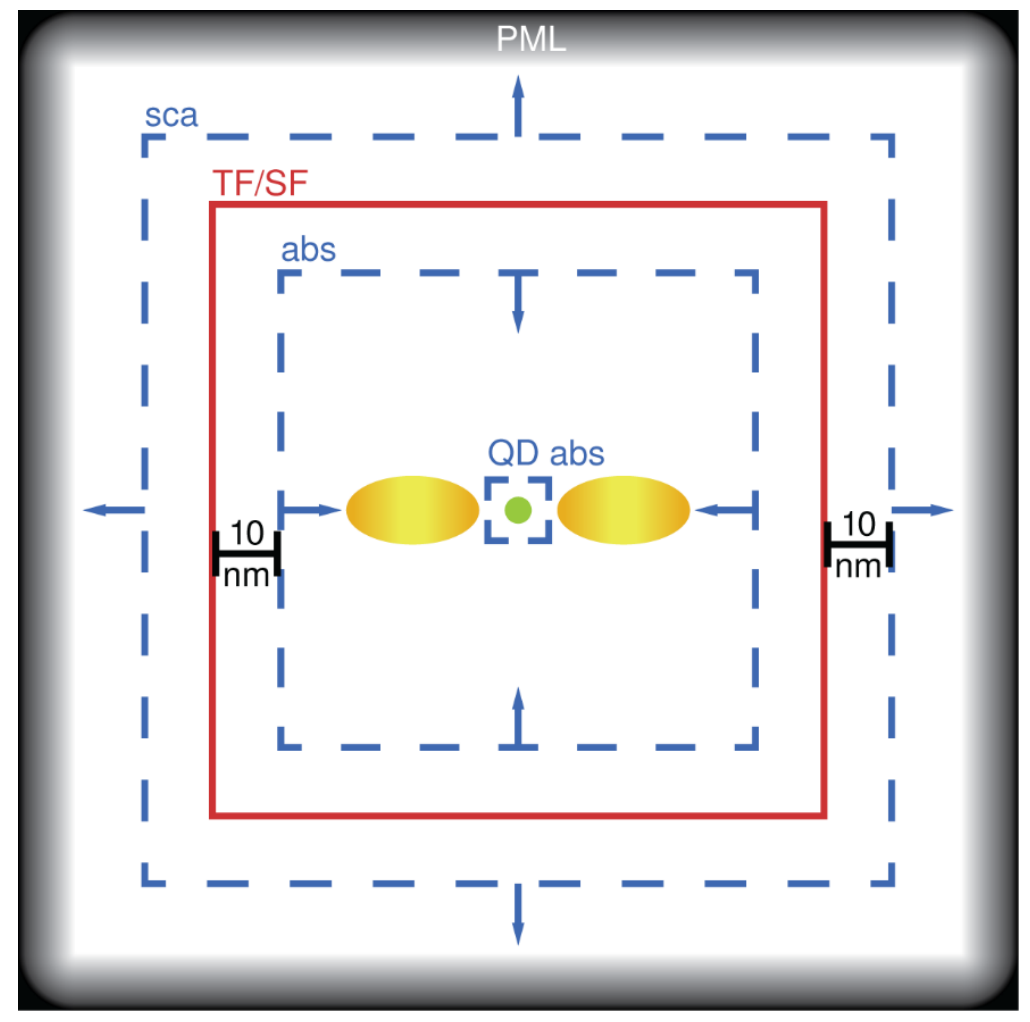

Figure S2: Diagram of the computational cell. The QD abs region corresponds to a flux region calculating only the quantum dot absorption, with the same directionality as the absorption flux.

\section{Gold Nanoisland film Model}

The gold nanoislands are modeled as a set of non-interacting, symmetric, elliptical nanodimers. The elliptical shape was chosen to best match the cross-section of the nanoislands. Figure S3 shows a schematic describing all tested models: monomers, symmetric dimers, and asymmetric dimers. The gap size for all the calculations was set to $7 \mathrm{~nm}$ or $10 \mathrm{~nm}$, representative of the range of likely QD sites in the films. $7 \mathrm{~nm}$ was chosen as the lower limit because that is the smallest size that can support a QD.

To match the experimental set up, the QD locations were randomized with the number of QDs set via a density parameter. To place each of the QDs in a cell, a random value for the $\mathrm{x}$ coordinate was selected and the y coordinate set such that the center of the QD is within $4 \mathrm{~nm}$ of the surface 
of the gold nanoparticles. Once placed, the QD was compared with previously placed QDs to avoid overlap of two QDs. This was repeated until all the QDs were placed in the cell.
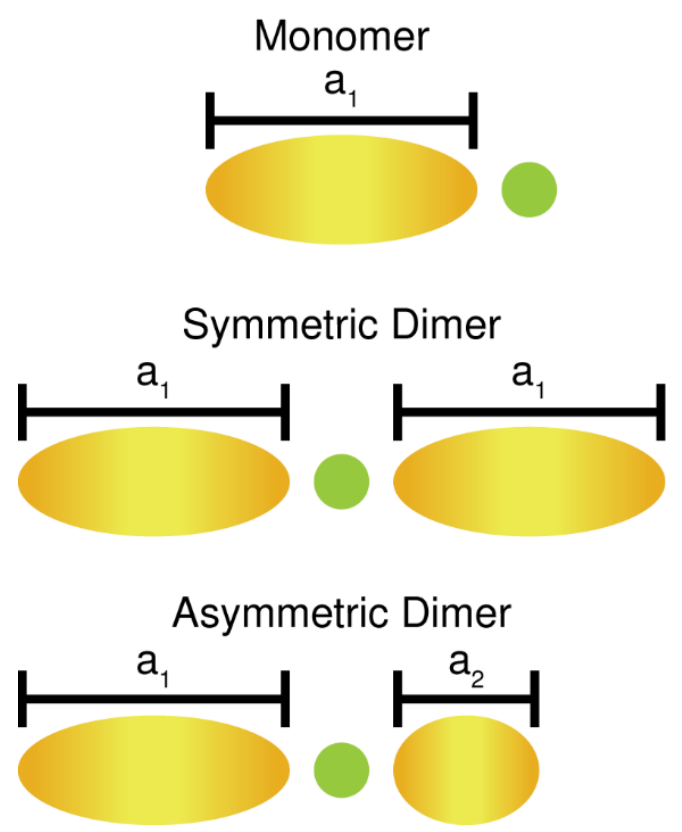

Figure S3: An illustration of all three tested nanoisland film models.

\section{Trimer confirmation}

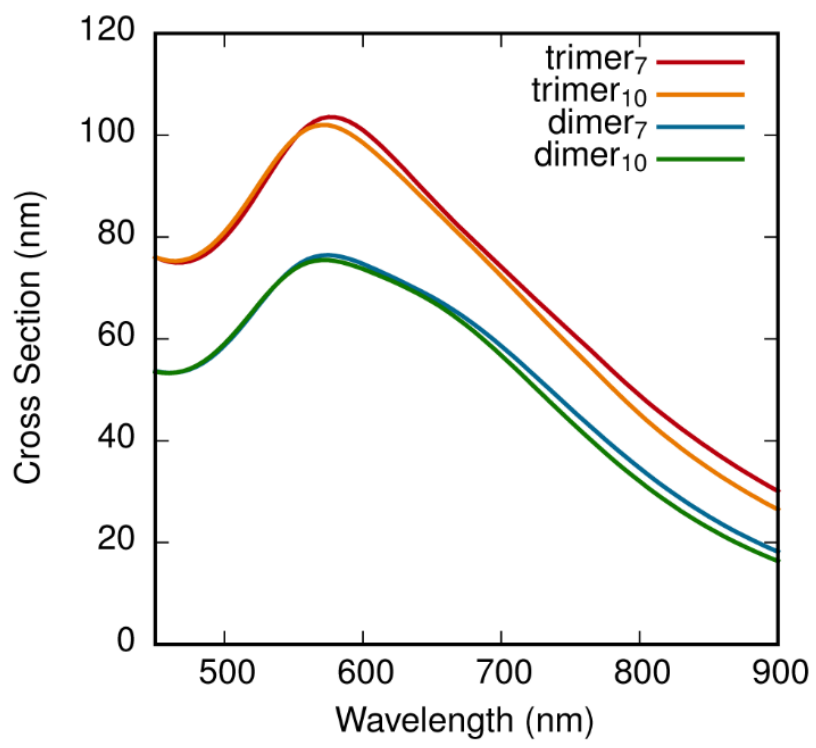

Figure S4: The absorption cross-section of the dimer (blue and green) and trimer (red and orange) gold nanoisland models. The subscript represents the gap size for each nanoisland system.

Figure S4 shows a comparison of the symmetric trimer calculations with the symmetric dimers. The peaks are in the same position for both the $10 \mathrm{~nm}$ and $7 \mathrm{~nm}$ gap, with the only difference 
being an increase in the absorption cross-section as a third particle is added and a slight decrease in the shoulder of the peak.

\section{Origin of the Dimer Blue-Shift}

The origin of the blue-shift in the dimer's absorption cross-section is explored in Figure S5, which shows the absorption cross-section for $200 \mathrm{~nm}$ long nanoellipse monomer and dimer. The monomer's absorption cross-section in Figure S5 a has a single peak at $862 \mathrm{~nm}$, while the dimer has two peaks at 1,008 $\mathrm{nm}$ and $658 \mathrm{~nm}$. The field maps in Figure S5 b show that the $658 \mathrm{~nm}$ peak is the result of the quadrupole resonance. The blue-shift is thus a result of averaging the quadrupole resonance and dipole resonance for dimers of different sizes.
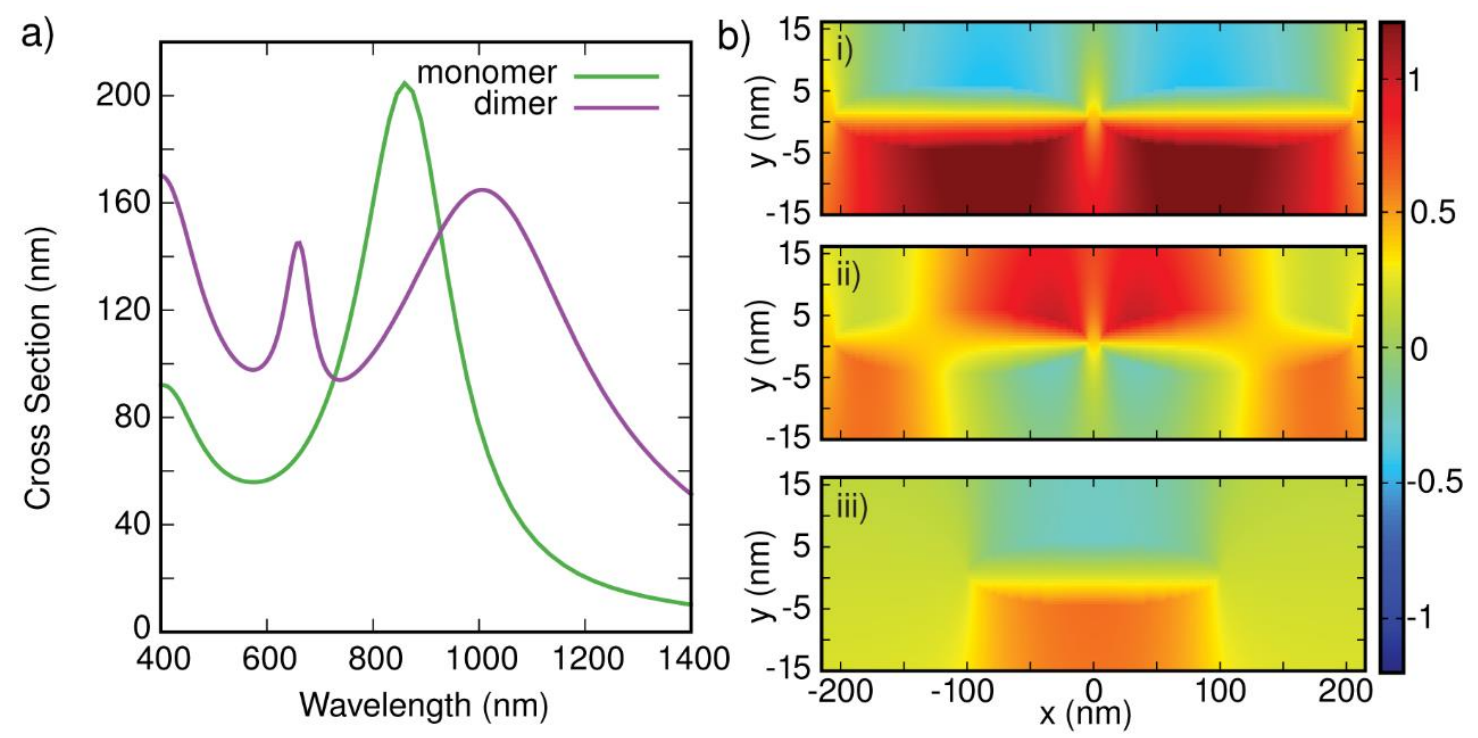

Figure S5: a) Simulated absorption cross-section for $200 \mathrm{~nm}$ by $11 \mathrm{~nm}$ gold nanoellipse monomer and dimer b) $\mathrm{H}_{\mathrm{z}}$ field map of the i) dimer excited at $658 \mathrm{~nm}$ ii) dimer excited at 1,008 $\mathrm{nm}$, and iii) monomer excited at $862 \mathrm{~nm}$.

\section{Plasmonic Loss}

The change in the gold nanoisland absorption after coupling to the QDs is highly dependent on the structural parameters of the system, as seen in Figure S6. The plots reinforce the message of Figure 3a that changes in the gold nanoisland absorption is the primary cause of the off-resonant peaks in the difference spectra and can make a significant contribution to the system's overall 
absorption. For the $110 \mathrm{~nm}$ nanoislands, the large negative values at wavelengths less than $\sim 750$ $\mathrm{nm}$ is the result of the red-shift of the plasmon resonance.
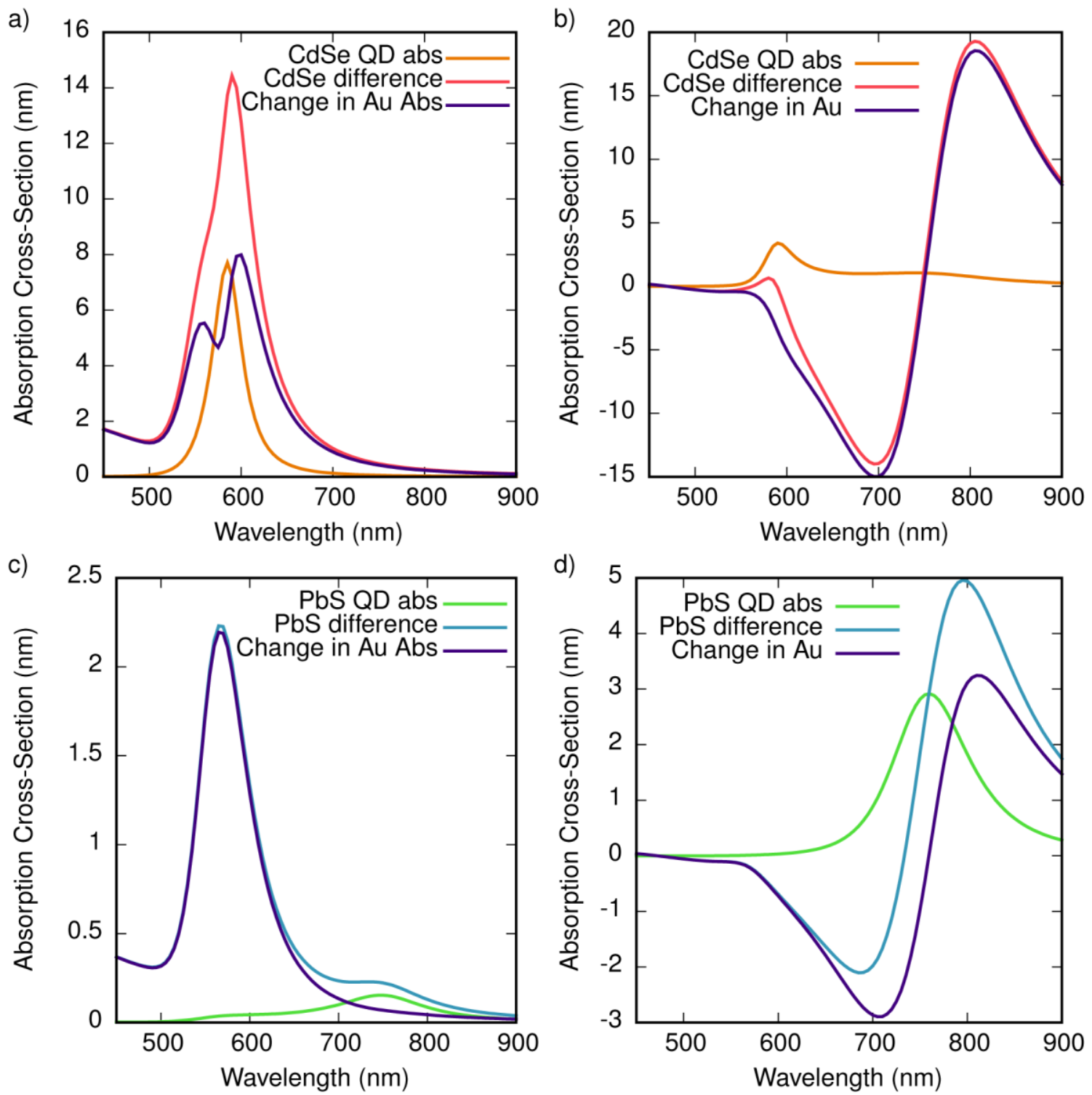

Figure S6: An approximation to the plasmonic loss into the $40 \mathrm{~nm}$ (a and c) and $110 \mathrm{~nm}(\mathrm{~b}$ and d) long dimers coupled to both the CdSe $610 \mathrm{~nm}$ QD (a and b) and PbS $850 \mathrm{~nm}$ QD (c and d) inside a $10 \mathrm{~nm}$ gap. The orange and green lines are the quantum dot absorption while the orange and blue lines are the difference between the gold nanoislands with and without the quantum dots. The purple lines represent the approximate change in the gold nanoisland's absorption cross-section. 


\section{Convergence of Peak locations and height for Density calculations}

Figure S7 shows the convergence patterns of the average peak wavelength and average peak absorption for different QD densities. All mean wavelengths and cross-sections were taken after 100 runs.

a)

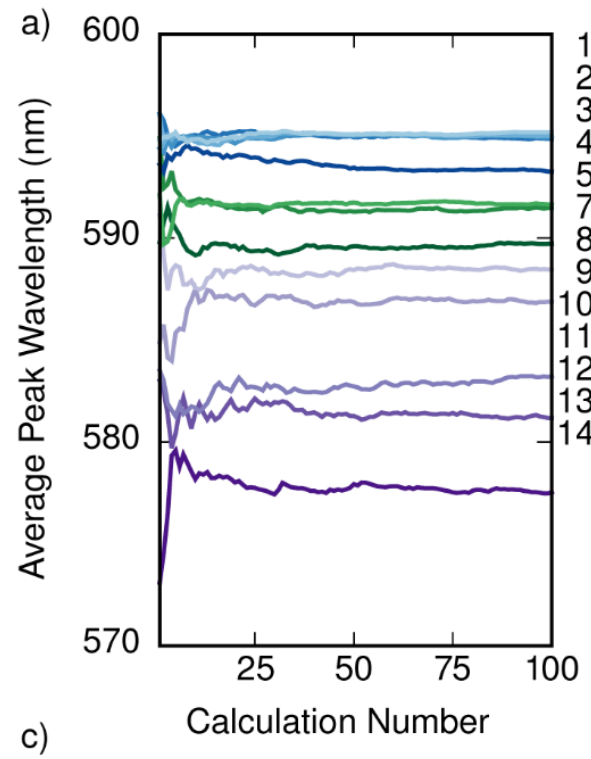

c)

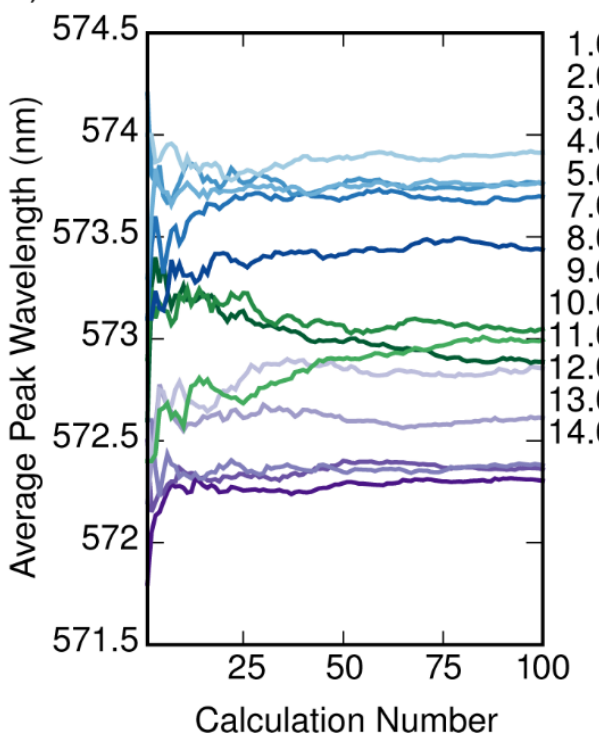

b)

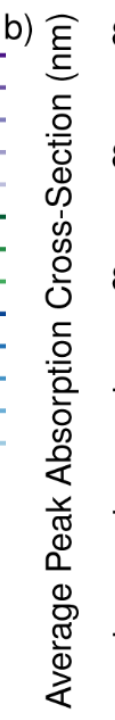

d)

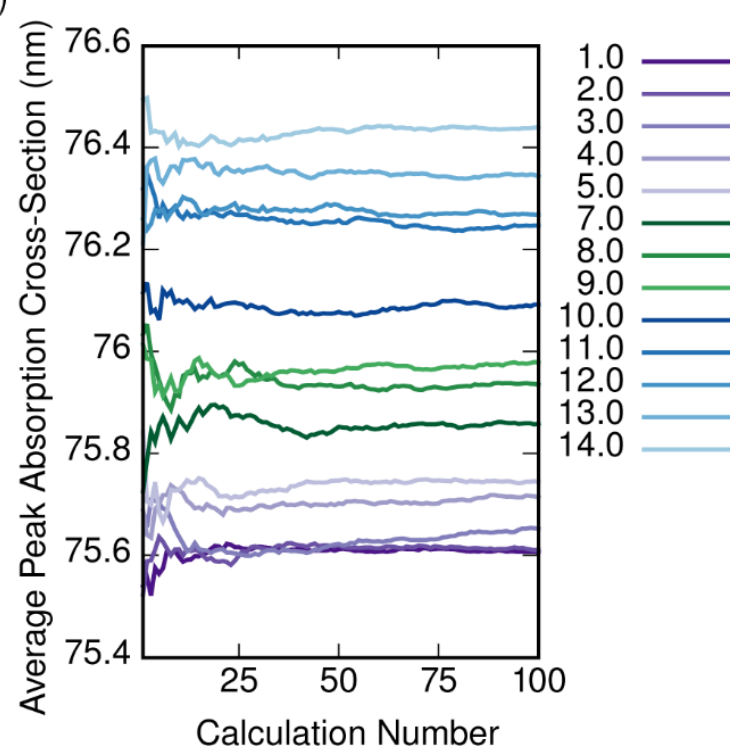

Figure S7: Convergence of the density calculations for the CdSe $610 \mathrm{~nm}$ QD (a and b) and the $\mathrm{PbS} 850 \mathrm{~nm}$ QD (c and d). The legends are in QDs/100 nm.

\section{Random Au-NPs preparation:}

Glass substrates were cleaned in Acetone and Ethanol. Electron gun evaporation method was use to evaporate ultrathin Au film of $5 \mathrm{~nm}$ nominal thickness. The deposition rate was $0.3 \AA / \mathrm{s}$ and it was done at room temperature under pressure of $\sim 10^{-6}$ torr. Samples were annealed on hot plate 
in air for several minutes. We used a simple hot plate (no thermometer included) reaching up to $\sim 200{ }^{\circ} \mathrm{C}$.

Monolayer preparation and QDs adsorption:

To form the monolayer of linker molecules the samples were left in absolute ethanol for 20 min, and then immersed for 17 hours in a $1 \mathrm{mM}$ 1,9 Nonanedithiol Ethanol solution under nitrogen environment. Subsequently the samples were washed in absolute Ethanol and Toluene solutions, immersed in a diluted $0.05 \%$ ww QD solution for another 17 hours and washed in absolute Toluene again. We used 2 types of commercial QDs: CdSe 610nm and PbS 850nm (the numbers represent the peaks of the corresponding emission wavelengths).

Only half of the sample was immersed in the solution in order to form a differential sample: The half without the dots was used as a reference in each sample.

Optical setup:

Total transmission and reflection were measured using Tungsten-Halogen lamp as a white light source, and an integrating sphere was used to overcome scatterings and achieve total transmission and reflection spectra. Data was recorded with Ocean Optics usb4000 VIS-NIR spectrometer. 